\title{
Behavioral Sensitivity to Broadband Binaural Localization Cues in the Ferret
}

\author{
Peter Keating, ${ }^{1}$ Fernando R. Nodal, ${ }^{1}$ Kohilan Gananandan, ${ }^{1}$ Andreas L. Schulz, ${ }^{1,2}$ \\ AND ANDREW J. King ${ }^{1}$ \\ ${ }^{1}$ Department of Physiology, Anatomy and Genetics, University of Oxford, Parks Road, Oxford, OX1 3PT, UK \\ ${ }^{2}$ Present Address: Leibniz Institute for Neurobiology, Brenneckestraße 6, 39118, Magdeburg, Germany
}

Received: 12 January 2013; Accepted: 5 April 2013; Online publication: 25 April 2013

\begin{abstract}
Although the ferret has become an important model species for studying both fundamental and clinical aspects of spatial hearing, previous behavioral work has focused on studies of sound localization and spatial release from masking in the free field. This makes it difficult to tease apart the role played by different spatial cues. In humans and other species, interaural time differences (ITDs) and interaural level differences (ILDs) play a critical role in sound localization in the azimuthal plane and also facilitate sound source separation in noisy environments. In this study, we used a range of broadband noise stimuli presented via customized earphones to measure ITD and ILD sensitivity in the ferret. Our behavioral data show that ferrets are extremely sensitive to changes in either binaural cue, with levels of performance approximating that found in humans. The measured thresholds were relatively stable despite extensive and prolonged (>16 weeks) testing on ITD and ILD tasks with broadband stimuli. For both cues, sensitivity was reduced at shorter durations. In addition, subtle effects of changing the stimulus envelope were observed on ITD, but not ILD, thresholds. Sensitivity to these cues also differed in other ways. Whereas ILD sensitivity was unaffected by changes in average binaural level or interaural correlation, the same manipulations produced much larger effects on ITD sensitivity, with thresholds declining when either of these parameters was reduced. The binaural sensitivity measured in this study can largely account for the ability of

\footnotetext{
Correspondence to: Andrew J. King • Department of Physiology, Anatomy and Genetics • University of Oxford • Parks Road, Oxford, OX1 3PT, UK. Telephone: +44-1865-272523; email: andrew.king@dpag.ox.ac.uk; Peter Keating - Department of Physiology, Anatomy and Genetics •
} University of Oxford - Parks Road, Oxford, OX1 3PT, UK. Telephone: +44-1865-282558; email: peter.keating@dpag.ox.ac.uk
\end{abstract}

ferrets to localize broadband stimuli in the azimuthal plane. Our results are also broadly consistent with data from humans and confirm the ferret as an excellent experimental model for studying spatial hearing.

Keywords: sound localization, spatial hearing, psychometric function, interaural time difference, interaural level difference, azimuth

\section{INTRODUCTION}

Over the past few decades, the ferret has emerged as an excellent model for studying the neural mechanisms underlying sound localization. Like humans, ferrets have good low-frequency hearing (Kelly et al. 1986) and possess immobile pinnae that filter sounds in a direction-dependent manner (Schnupp et al. 2003). Given their relative immaturity at birth, ferrets are also highly suitable for studying the role of sensory experience in the development of sound localization mechanisms (King and Parsons 1999; Moore 2002; King et al. 2011). In addition, the temperament of this species makes it particularly useful for behavioral assessments of spatial (Kavanagh and Kelly 1987; Hine et al. 1994; Nodal et al. 2008) as well as other aspects (Kelly et al. 1996; Walker et al. 2009; Yin et al. 2010; Alves-Pinto et al. 2012; Bizley et al. 2013) of hearing.

More recently, the ferret has also been developed as an experimental model for cochlear implantation (Hartley et al. 2010). In part, this work is motivated by questions concerning the clinical benefits of bilateral cochlear implantation. One key issue concerns the factors that help bilateral implantees to take advantage of binaural spatial cues. Because sensitivity to 
interaural time differences (ITDs) and interaural level differences (ILDs) is critical for sound localization and sound source separation in noisy environments (Hine et al. 1994; Roman et al. 2003; Bee and Micheyl 2008), understanding how these binaural spatial cues are processed is of clear interest both from a clinical and basic science perspective.

Previous studies of sound localization behavior in the ferret have presented stimuli via free-field speakers situated distal to the animal. Whilst these studies have revealed how well ferrets can localize sounds in azimuth (Kavanagh and Kelly 1987; Parsons et al. 1999; Nodal et al. 2008) and elevation (Parsons et al. 1999; Bizley et al. 2007), little is known about the contributions of different auditory spatial cues to these tasks. Reversible occlusion of one ear has been used to demonstrate the importance of binaural cues in azimuthal localization (Kacelnik et al. 2006), while manipulations of the pinnae have revealed the role of spectral cues (Parsons et al. 1999; Kacelnik et al. 2006). The only previous attempt, however, to dissociate the contribution made by ITDs and ILDs employed narrowband stimuli with different center frequencies (Kacelnik et al. 2006). Assuming that lowand high-frequency sounds are respectively localized on the basis of ITDs and ILDs, this work suggests that ferrets rely on both of these cues. This is consistent with neurophysiological studies in this species, which have used closed-field techniques to demonstrate neural sensitivity to ITDs and ILDs (Campbell et al. 2006; Hartley et al. 2011).

Whilst these results suggest that ferrets can distinguish behaviorally between different ITD and ILD values, these abilities have yet to be directly tested. In the present study, we used closed-field techniques to measure the behavioral sensitivity of ferrets to these cues. As in most of the earlier free-field studies, we focused on broadband stimuli and examined the effects of variations in stimulus envelope, level, duration, and interaural correlation on the animals' sensitivity to ITDs and ILDs.

\section{METHODS}

Five adult pigmented female ferrets (Mustela putorius) from our breeding colony were used for the purposes of this study. All procedures were performed under licenses granted by the UK Home Office and met with ethical standards approved by the University of Oxford. Each period of behavioral testing lasted for a maximum of 14 days, during which drinking water was provided through correct performance of the task and additional supplements provided at the end of each day. Animal weights were monitored routinely, with any substantial reductions in weight $(>2 \mathrm{SD}$ below the mean) resulting in the termination of a particular testing period. Dry food was available at all times and animals were given free access to water between testing periods.

\section{Behavioral apparatus}

Behavioral testing was performed in a standard mesh cage $(55 \times 43 \times 41 \mathrm{~cm})$ with a solid plastic floor, which was enclosed with acoustic foam (MelaTech; Hodgson \& Hodgson Ltd., Melton Mowbray, UK). The animal initiated a trial by inserting its nose into a poke-hole located in the middle of the front wall of the cage. After a variable delay, a stimulus was presented and the animal responded by inserting its nose into pokeholes located on either side of the testing cage (Fig. 1A). Infrared LEDs were used to produce beams of light spanning the entrance to each poke-hole, the status of which was monitored continuously by photodiodes connected to a real-time processor (RP2; Tucker-Davis Technologies, Alachua, FL). Poking behavior readily interrupted these beams, enabling responses to be registered. Correct responses were rewarded with a specified amount of water (typically $150-300 \mu \mathrm{L}$ per trial) that was delivered via spouts situated in each of the poke-holes. Incorrect responses were followed by correction trials on which the same stimulus was presented, with these correction trials continuing until the animal responded correctly.

All electronic circuits were constructed using standard components (RS Components, Corby, UK) according to a design similar to that described in Walker et al. (2009) and all experimental contingencies were controlled using Matlab (The Mathworks, Natick, MA). Stimuli were also generated in Matlab and then sent to an RP2 via a USB2 interface. Following amplification, these signals were presented to the animal either via loudspeakers (FRS 8; Visaton, Crewe) located on either side of the testing cage or via earphones (RP-HV280, Panasonic, Bracknell, UK). Earphone cables exited through the ceiling of the cage and were subject to a minimal amount of tension to ensure that they did not interfere with the movement of the animal.

\section{Closed-field sound delivery system}

Earphones were attached to lightweight titanium holders $(11.5 \mathrm{~g})$ that were in turn secured using bolts to an implant that had previously been mounted on the head of the animal under anesthesia. The design of both the implant and earphone holders, as well as the surgical details involved in implantation, have been detailed elsewhere (Nodal et al. 2010) and so are only briefly described here. Earphone holders were 
A

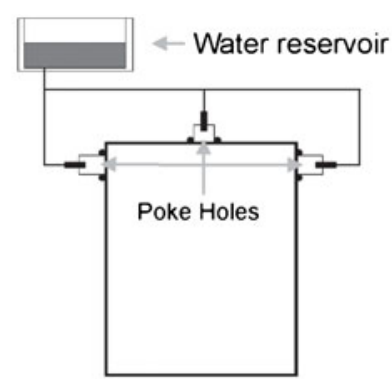

C
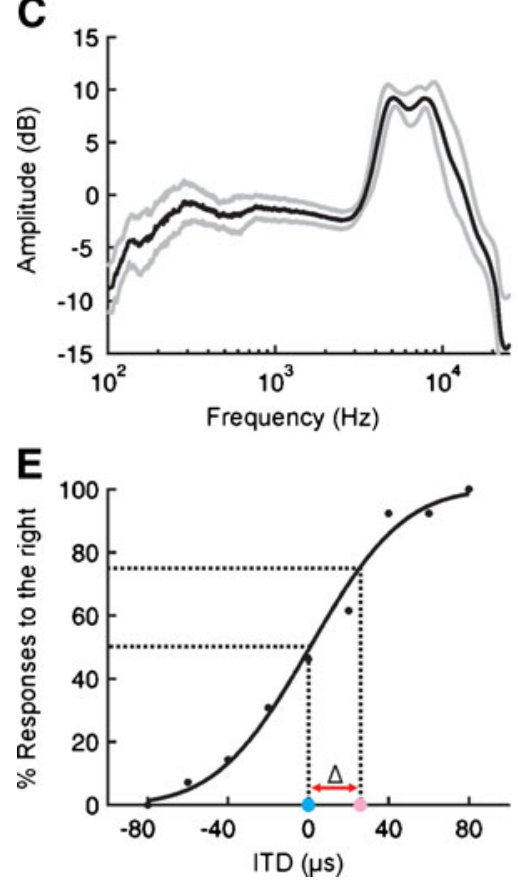

B
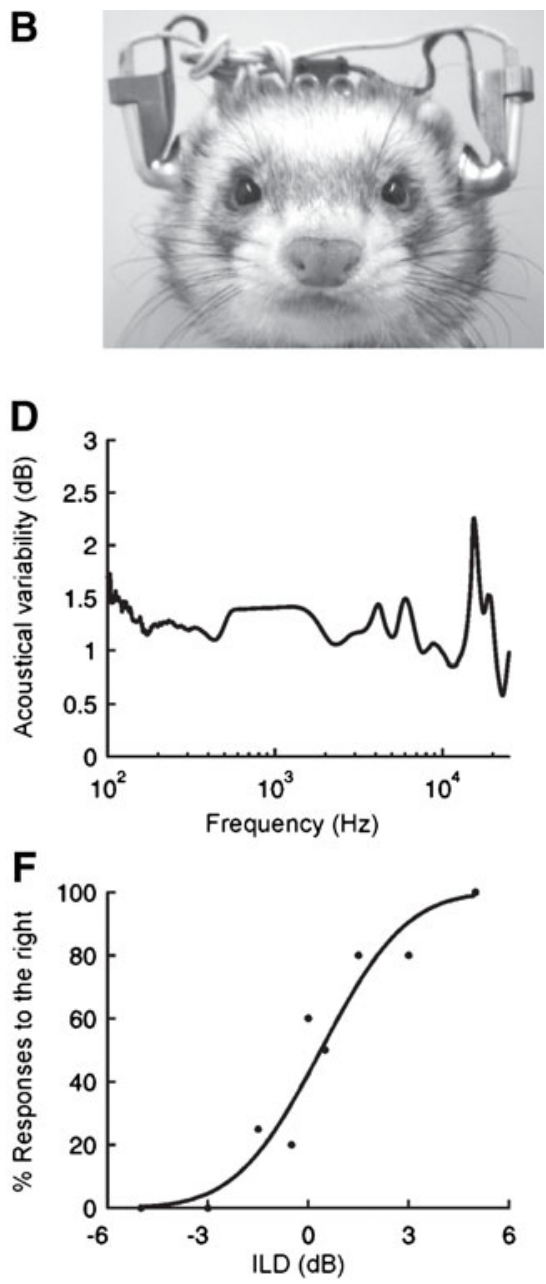

FIG. 1. Behavioral methods. A Schematic of setup used for behavioral testing. Each animal initiated a trial by poking its nose into a central poke-hole. A stimulus was then presented via head-mounted earphones and the animal registered its response via the poke-holes located on either side of the testing chamber. Correct responses were rewarded with a small amount of water. B Illustration of ferret wearing earphones. C Uncorrected transfer function (mean $\pm \mathrm{SD}$ ) of the earphone drivers used. D Variability (SD) in the amplitude spectrum of the same stimuli presented repeatedly after detaching and reattaching earphones. E Sample psychometric function for the ITD task obtained during a single session. Percentage of trials on which the animal responded at the right poke-hole is plotted as a function of ITD. Filled black circles show raw data, with the solid black line displaying the best fit line estimated using probit analysis. Threshold $(\Delta)$ was defined by calculating the difference (red line) between ITD values that elicited responses to the right on $50 \%$ (blue circle) and $75 \%$ (pink circle) of trials. F Sample psychometric function for the ILD task. designed so that they could be easily disconnected and reattached to the implant, which made it possible to remove the earphones and their holders whenever the animal was not performing the behavioral task. The holders were also adjustable, allowing the earphones to be positioned consistently just in front of the ear canals of each animal (Fig. 1B).

The cranial implant consisted of two bolts that were encased in CMW1 bone cement (DePuy CMW, Lancashire, UK) and attached directly to the skull using dental adhesive (Super-bond C\&B; Sun Medical Co, Shiga Japan). Prior to implantation under sterile conditions, animals were anesthetized by intramuscular administration of $0.022 \mathrm{mg} / \mathrm{kg}$ medetomidine hydrochloride (Domitor; Pfizer Ltd., Kent, UK) and $5 \mathrm{mg} / \mathrm{kg}$ ketamine (Ketaset; Fort Dodge Animal Health, Southampton, UK). The left radial vein was then cannulated for administration of drugs and fluid, which was followed by intubation and mechanical ventilation, thereby allowing the anesthetic agent to be switched to $0.5-1.5 \%$ isoflurane (IsoFlo; Abbott Laboratories Ltd., Kent, UK). This was followed by intramuscular administration of atipamezole (Antisedan; Pfizer Animal Health, Kent, UK) to reverse the effects of medetomidine, atropine (0.006 mg/kg Atrocare; Animalcare Ltd., York, UK) to reduce secretions, and buprenorphine $(0.03 \mathrm{mg} /$ kg Vetergesic; Alstoe Animal Health, Melton Mowbray, UK) and meloxicam $(0.2 \mathrm{mg} / \mathrm{kg}$ Metacam; Boehringer Ingelheim, Terrassa, Spain) to provide perisurgical analgesia. Local anesthetic (Elma; Astra Zeneca Luton, UK) was then applied to stereotaxic pressure points and carbomer (Viscotears; Lewis Pharmaceuticals Ltd., Doncaster, UK) was applied to the eyes.

In order to construct the implant, the head was positioned in a stereotaxic frame, a midline incision was used to expose the dorsal part of the skull, and the temporal muscles were displaced laterally. The skull was then cleaned using a $1 \%$ citric acid solution, following which the base of the implant was attached to the skull, and then progressively built up using layers of bone cement. ECG and end tidal $\mathrm{CO}_{2}$ were monitored throughout. Once complete, the skin and temporal muscles were repositioned and sutured together to the front and rear of the implant. The earphone holders were then attached and adjusted so that the earphones were situated correctly with 
respect to the ears (Fig. 1B), with each animal receiving its own individualized holder. Following recovery from anesthesia, the animal was returned to its cage and given buprenorphine for 3 days, and meloxicam for 5 days, after implantation. The animal was then allowed to recover completely prior to behavioral testing.

\section{Acoustical measurements}

Acoustical measurements were carried out in an anechoic chamber (Industrial Acoustics Ltd., Winchester, UK) using a ferret cadaver that underwent the same implantation procedure as that adopted for the animals that were used for behavioral testing. The ferret cadaver was placed at the center of the anechoic chamber and polythene tubes (o.d. $1.52 \mathrm{~mm}$, i.d. $0.86 \mathrm{~mm}$ ) were inserted at the entrance of each ear canal and attached to a pair of Sennheiser microphone capsules (KE-4-2112; Wedemark-Wennebostel, Germany) via customized connectors. These were then used to record acoustic responses at each ear.

Although the earphone drivers used in this study were very similar to one another, they had a distinctive spectrum that varied by $\sim 20 \mathrm{~dB}$ at different frequencies (Fig. 1C). Prior to any acoustical measurements, the transfer function of each earphone was therefore measured, flattened, and calibrated to $85 \mathrm{~dB}$ SPL. For the purposes of acoustical testing, all stimuli were generated and recorded using an RP2 running at $97.6 \mathrm{kHz}$, controlled via customized Matlab code. Twenty 1,000-ms bursts of broadband noise were then presented, with the earphones detached and reattached between each presentation. This enabled us to estimate the replicability of acoustical conditions across different behavioral sessions. Because the earphone holders were attached to the implant using two bolts, they were relatively stable under conditions that approximated normal use, with the standard deviation of these measurements typically $<1.5 \mathrm{~dB}$ (Fig. 1D).

\section{Behavioral testing}

Prior to attaching the head implant, animals were trained by positive reinforcement to perform a twoalternative, forced-choice task using free-field speakers located on either side of the testing cage. During the initial training, the animal had to insert its nose into the center spout poke-hole for $200 \mathrm{~ms}$ after which a sound was played from one of the speakers. In order to receive a reward, the animal had to approach the source of the sound and place its nose into the underlying poke-hole containing a spout.

Once the animal had learned the mechanics of this task and was performing $\sim 100$ trials on each session, we gradually increased the period over which it had to remain at the center spout prior to trial initiation and allowed this delay to vary from 1-7 s. Identical stimuli were then presented over both speakers simultaneously, but with a $30-\mathrm{dB}$ difference in level between them, with the animal required to indicate the location of the sound by poking its nose into the poke-hole on the side of the more intense sound. To avoid the animal developing a bias towards one response location, an incorrect response was followed by a number of correction trials (same interspeaker level difference) until it responded correctly. Once the animals attained a stable performance of $>97 \%$ correct for several days, they were judged to be adequately trained and ready for implantation of the cranial support. In total, this period of training took 1-3 weeks depending on the animal.

After implantation, animals were again tested to confirm that they could still discriminate between the same free-field noise bursts. The earphone holder was then attached so that broadband stimuli could be presented over earphones. Once the animals were able to reliably achieve a performance level over several days of $>97 \%$ correct with an ILD of $\pm 15 \mathrm{~dB}$, the ILD or ITD was varied so that psychometric functions could be derived.

Each animal was required to perform an ILD task and an ITD task, both of which used the method of constant stimuli. For the ITD task, the ILD was set to 0 and ITDs were allowed to vary in $20 \mu$ s steps between -80 and $80 \mu \mathrm{s}$, where negative values denote stimuli that favored the left ear. ITDs were imposed by delaying the entire waveform of the signal presented to one ear relative to the other and, within a single session, all ITD stimuli were presented at the same level. The ITD was set to $0 \mu \mathrm{s}$ for the ILD task, with ILDs chosen from the set: $\{-5,-3$, $-1.5,-0.5,0,0.5,1.5,3,5 \mathrm{~dB}\}$. ILDs were imposed by adjusting the levels in each ear in such a way that the average binaural level (ABL) was kept constant. To ensure that animals could not perform the task using information about monaural level, the ABL was allowed to vary randomly by up to $10 \mathrm{~dB}$ across trials. The animals typically completed these tasks in blocks, with the sessions contained in each block all requiring the animal to lateralize on the basis of the same cue. In the small number of cases where thresholds could not be estimated using the values described above, values were scaled by a factor of 2 or 3 to assess whether thresholds could be estimated using larger ITDs or ILDs. If thresholds could not be estimated with these larger values, we report thresholds as being immeasurable.

\section{Stimuli}

To investigate the effects of different stimulus parameters on binaural cue sensitivity, a number of different stimuli were used throughout this study. In an initial 
set of experiments, we used broadband noise, the spectrum of which was entirely determined by both the Nyquist frequency $(24.4 \mathrm{kHz})$ and the transfer function of the earphones used (Fig. 1C). For these stimuli, no attempt was made to flatten the transfer function of the earphones, which was highly conserved across different earphone drivers. Subsequent experiments, however, obtained similar thresholds with noise that was band-pass filtered and flattened from 0.5 to $20 \mathrm{kHz}$. ITD thresholds obtained with the two sets of noise stimuli did not differ significantly $(F(1,144)=0.84, P=0.36)$, whereas ILD thresholds were slightly lower $(\sim 0.5 \mathrm{~dB})$ using the uncorrected transfer function of the earphone drivers $(F(1,131)=$ 12.93, $P=0.0005)$. This is most likely because the energy in these stimuli was shifted toward higher frequencies, which would be expected to improve ILD sensitivity (Witten et al. 2010). In measuring the sensitivity of ferrets to binaural spatial cues, we did not convolve our broadband stimuli with the headrelated transfer function of the animals.

To investigate the effects of envelope, we added $75 \mathrm{~Hz}$ sinusoidal amplitude modulation (SAM, with a modulation depth of $100 \%$ ) to broadband noise. Unmodulated noise was additionally used to estimate the effects of level and interaural correlation. However, whereas $10-\mathrm{ms}$ cosine ramps were used for the majority of stimuli in this study, and were gated independently for each ear, the effects of level and interaural correlation were measured using 50-ms cosine ramps that were gated simultaneously for the two ears.

Interaural correlation was controlled using a relatively simple method, which compared favorably with more complex methods (Culling et al. 2001) for the levels of correlation used in this study. In particular, the signal in any given ear $\left(S_{\mathrm{i}}\right)$ was created from the weighted linear combination of two signals $\left(S_{1}, S_{2}\right)$, one of which was shared between the two ears and one of which was unique to each ear:

$$
S_{\mathrm{i}}=w_{\rho} S_{1}+\left(1-w_{\rho}\right) S_{2}
$$

where $w_{\rho}$ represents the weighting factor that produces a level of interaural correlation equal to $\rho$. Appropriate weighting factors were determined numerically for each level of correlation used. Throughout the entire study, all stimuli were generated in Matlab and presented at a sampling rate of $48.8 \mathrm{kHz}$.

Animals were initially trained on unmodulated noise stimuli and then, in the following order, tested on (1) unmodulated noise, (2) sinusoidal amplitude modulated noise, and (3) unmodulated noise stimuli. For each stimulus type, animals completed a block of ILD sessions followed by a block of ITD sessions (except for one animal which did the opposite, but this did not influence the results in an obvious way). Within each block, animals first completed three to six consecutive sessions using the longest duration stimuli (200 ms). Once an adequate number of sessions had been completed satisfactorily, each animal then completed a similar number of sessions for the next longest duration (100 ms), before repeating this process for the shortest duration tested (40 ms). For this initial phase of testing, all stimuli were presented at an ABL of $75 \mathrm{~dB}$ SPL.

Once this phase of testing was complete, we then used 100-ms unmodulated noise stimuli to (1) explore the effects of level before (2) investigating the effects of interaural correlation. As before, animals performed ITD and ILD tasks in separate blocks and were initially tested on either the loudest or most correlated stimuli before moving on to stimuli that were progressively more quiet or less correlated, respectively.

\section{Statistical analysis}

Because subjects were repeatedly tested in multiple sessions using the same task and stimulus, statistical tests of significance were generally conducted on thresholds obtained from individual sessions using mixed-effects ANOVAs with subject as a random factor. In all cases, post hoc analyses were performed using Tukey's HSD to correct for multiple comparisons.

\section{RESULTS}

In order to measure behavioral sensitivity to binaural spatial cues, we presented broadband stimuli to ferrets over earphones and varied either the ITD or the ILD. Ferrets were required to lateralize these stimuli and respond to the correct side in order to obtain a reward. These responses were then used to construct psychometric functions (Fig. 1E, F), which show the percentage of responses made to the right as a function of either ITD or ILD. Using probit analysis, these data could be well approximated by a sigmoidal function that consists of just two parameters.

The first of these measures the response bias of a subject and is determined by the cue value that elicits an equal proportion (i.e., $50 \%$ ) of responses to either side (see Fig. 1E). Our primary focus, however, was on the second parameter, which measures the sensitivity of a subject to changes in a particular cue. This second parameter is determined by the steepness of the psychometric function, with steeper functions associated with greater sensitivity. We quantified this by calculating a threshold $(\Delta$, Fig. $1 \mathrm{E})$, defined here as the difference between the cue value that elicited 
maximal uncertainty in the response (i.e., $50 \%$ responses to the right) and the value that elicited relatively reliable responses to a particular side (i.e. $75 \%$ responses to the right). This criterion was primarily adopted to facilitate comparison with other species (Zwislocki and Feldman 1956; Klumpp and Eady 1957; Mills 1960; Wakeford and Robinson 1974; Yost 1974; Scott et al. 2007), as well as with free field studies of spatial sensitivity in the ferret (Kavanagh and Kelly 1987; King and Parsons 1999), and corresponds to a $d^{\prime}$ value that is very close to 1 on a $2 \mathrm{AFC}$ task (Green and Swets 1966).

\section{ILD sensitivity}

Because many electrophysiological studies are performed in experimentally naïve animals, we were interested in measuring thresholds and response bias prior to extensive testing on the perceptually challenging aspects of the task. To the extent that task performance was improved by behavioral testing, we were also interested in quantifying the amount of learning that took place. For this reason, we trained animals on the procedural aspects of a lateralization task using ILD values that were considerably above threshold $(>15 \mathrm{~dB})$. We only measured thresholds when ferrets were required to lateralize perceptually difficult ILDs for the first time. During this initial test period, ILD thresholds were typically $<2 \mathrm{~dB}$ (Fig. 2A), with thresholds $<1 \mathrm{~dB}$ obtained for some animals during specific testing sessions.

To assess the stability of task performance, we retested these animals at least 16 weeks later using the same stimuli. In the intervening time, animals were extensively tested using both ITD and ILD tasks. Although this testing could have altered thresholds over time, performance during the second test period was very similar to that measured during the first test period (Fig. 2A). Using a mixed-effects ANOVA, with testing period and duration as fixed factors and subject as a random factor, we found no difference between the ILD thresholds obtained during these two testing periods $(F(1,84)=0.63, P=0.429)$, indicating that behavioral performance was relatively stable over time. We therefore pooled the data collected during these two testing periods for all subsequent analyses.

Between the two testing periods referred to above, animals were tested using the same stimuli, but with SAM added to the envelope, a change that failed to produce any obvious effect except for a marginal increase in threshold across all durations (Fig. 2B). The ILD thresholds obtained for each individual testing session were then submitted to a mixed-effects ANOVA, with envelope type and duration as fixed effects and subject as a random effect. This analysis revealed no effect of envelope type $(F(1,178)=0.01$, $P=0.91$ ), but did show a significant effect of duration $(F(2,178)=14.55, P<0.00001)$. Post hoc tests showed significant differences between the shortest duration and all other durations tested $(P<0.05)$. This reflected the fact that ILD thresholds were larger for all stimulus types at the shortest durations tested. In contrast, estimates of ILD response bias were unaffected by either envelope type $(F(1,178)=1.36, P=0.245)$ or duration $(F(2,178)=1.97, P=0.14)$. Moreover, although individual variations in bias were apparent, the mean bias across subjects and stimulus types was very close to 0 (Fig. 2C).

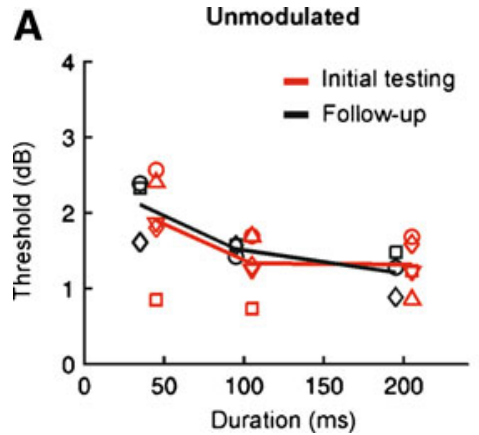

FIG. 2. Effect of stimulus duration and signal envelope on ILD sensitivity. A Unmodulated broadband noise. Mean thresholds across subject are plotted for an initial testing period (red line) as well as a second testing period (black line) completed following more than 16 weeks of ITD and ILD testing. Data for individual animals are denoted by symbols of the same color (each of these data points shows the mean threshold across at least three testing sessions). In the time between the two test periods shown, animals received extensive training on both ITD and ILD tasks using a variety of different stimuli. B Thresholds for
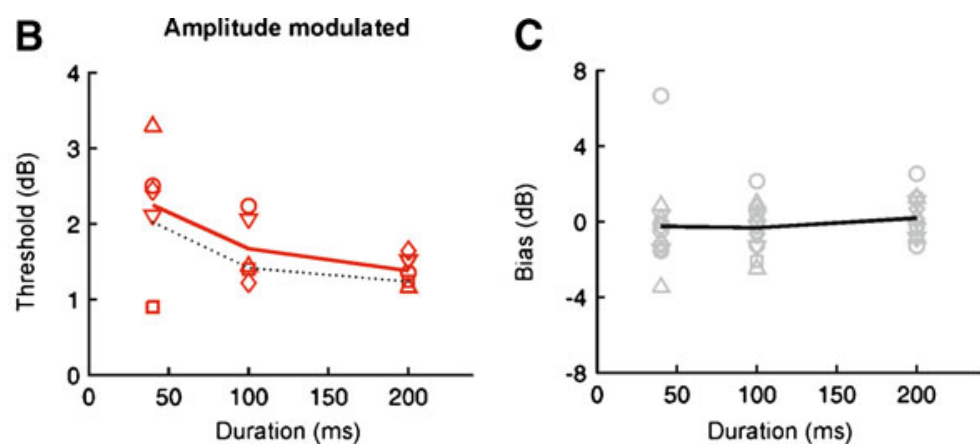

sinusoidally amplitude modulated noise. Continuous line shows mean thresholds across subjects; symbols show data for individual subjects. To aid comparison across envelope types, dotted lines show the mean of the two lines displayed in panel A. C Estimates of response bias collapsed across signal type. Each gray symbol shows data obtained for a single subject and signal type. The black line shows estimates of bias averaged across different subjects and signal types, and plotted as a function of duration. 


\section{ITD sensitivity}

Using the same stimuli and experimental approach, we also obtained estimates of ITD sensitivity. As before, thresholds were initially measured using broadband noise with a relatively flat envelope. These initial ITD thresholds were typically $<40 \mu$ s (Fig. $3 \mathrm{~A}$ ), but tended to improve at longer durations. Sixteen weeks later, ITD thresholds remained unchanged (Fig. 3A; $F(1,102)=$ $0.88, P=0.350)$, despite the fact that animals were extensively tested on both ITD and ILD tasks in the interim. The data for these two testing periods were therefore pooled for subsequent analyses.

We found that thresholds tended to increase very slightly following a change in the envelope of the stimulus and were also typically larger at the shortest durations tested (Fig. 3A, B). A mixed-effects ANOVA with envelope type and duration as fixed effects and subject as a random effect revealed significant main effects of both envelope type $(F(1,190)=6.75, P=0.01)$ and duration $(F(2,190)=32.77, P<0.00001)$ on the ITD thresholds. Post hoc analyses showed significant differences between the shortest duration and all other durations tested $(P<0.05)$. This reflects the fact that thresholds tended to be larger for all stimulus types at the shortest durations tested and were slightly higher overall when SAM was added to the envelope (Fig. 3A, B). As was the case for ILD performance, estimates of ITD response bias were unaffected by either stimulus type $(F(1,190)=0.06, P=0.81)$ or duration $(F(2,190)=$ 1.91, $P=0.15$; Fig. 3C).

\section{Effect of sound level}

We assessed the effects of varying sound level on ITD and ILD sensitivity using 100-ms bursts of flat-spectrum noise that were presented at an ABL of either 35 or $75 \mathrm{~dB}$ SPL. A mixed effects ANOVA, with ABL as a fixed effect and subject as a random effect, showed that ILD thresholds did not differ at these levels $(F(1,36)=0.07, P=0.7881$; Fig. 4 A $)$.
In contrast, ITD thresholds were significantly larger at $35 \mathrm{~dB}$ SPL relative to $75 \mathrm{~dB}$ SPL $(F(1,16)=12.41$, $P=0.0028$; Fig. $4 \mathrm{~B}$ ). This analysis is likely to underestimate the effect of ABL on ITD sensitivity, since one animal was completely unable to perform the ITD task at $35 \mathrm{~dB}$ SPL, and we were unable to obtain thresholds for two additional animals in $50 \%$ and $33 \%$ of sessions respectively (i.e., their performance was indistinguishable from chance, despite completing $>50$ trials). Because thresholds could not be estimated, these data were excluded, thereby biasing our estimates of threshold downwards.

Estimates of response bias were unaffected by ABL for both ILD $(F(1,36)=0.01, P=0.90$; Fig. $4 \mathrm{C})$ and ITD $(F(1,16)=1.42, P=0.25$; Fig. $4 \mathrm{D})$ tasks.

\section{Effect of interaural correlation}

ITD sensitivity is thought to depend on the degree of correlation between the acoustical signals provided to each ear (Blauert and Lindemann 1986; Saberi et al. 1998). ILD sensitivity, however, is thought to depend on alternative mechanisms that are independent of interaural correlation (Egnor 2001; Peña 2003). To address this issue in the ferret, we measured ITD and ILD thresholds using the same flat-spectrum noise stimuli employed to assess the effects of ABL, but with a fixed ABL of $75 \mathrm{~dB}$ SPL, and manipulated the degree of interaural correlation.

As predicted by data from other species, ILD thresholds remained essentially unchanged despite large changes in interaural correlation (Fig. 5A). This was confirmed by a mixed-effects ANOVA, with the degree of interaural correlation as a fixed effect and subject as a random effect, which showed no effect of interaural correlation on the estimated thresholds $(F(2,33)=1.15, P=0.3296)$. ITD thresholds, however, increased rapidly as interaural correlation was reduced (Fig. 5B). Indeed no ITD threshold could be estimated for any animal when the level of interaural
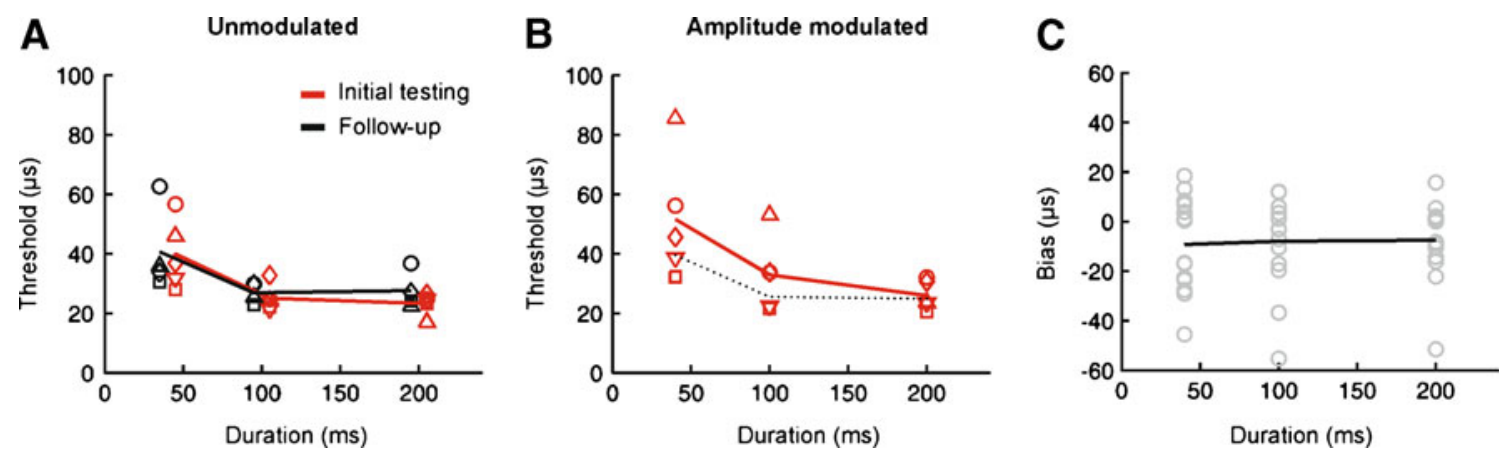

FIG. 3. Effect of stimulus duration and signal envelope on ITD sensitivity. Plotting conventions are identical to Fig. 2. A Unmodulated broadband noise. B Sinusoidally amplitude modulated noise. C Estimates of response bias collapsed across envelope type. 

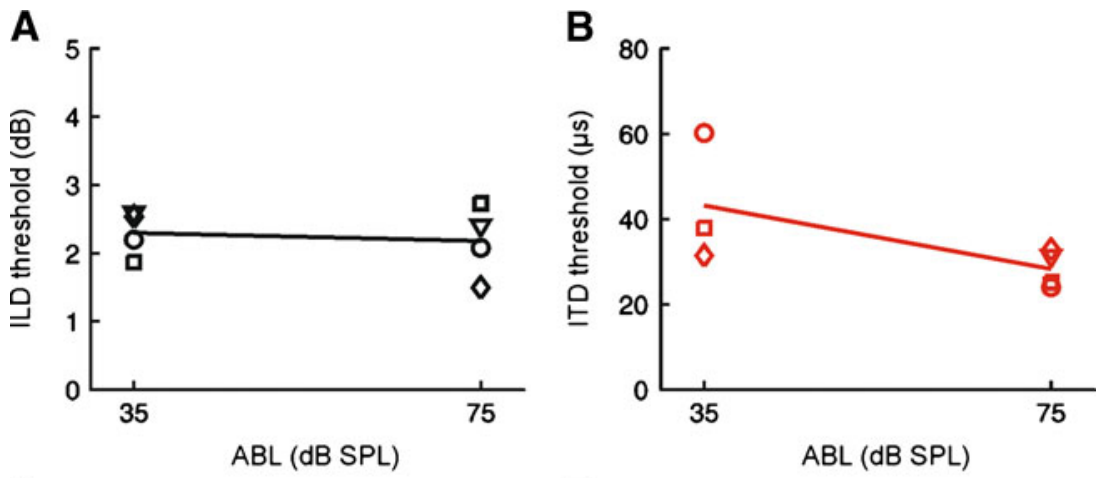

FIG. 4. Effect of ABL on ILD and ITD sensitivity. Symbols show data for individual animals, with lines showing the mean across subjects. $\mathbf{A}$ Effect of $A B L$ on ILD thresholds. B Effect of ABL on ITD thresholds. $\mathbf{C}$ Effect of ABL on ILD bias. D Effect of $A B L$ on ITD bias.
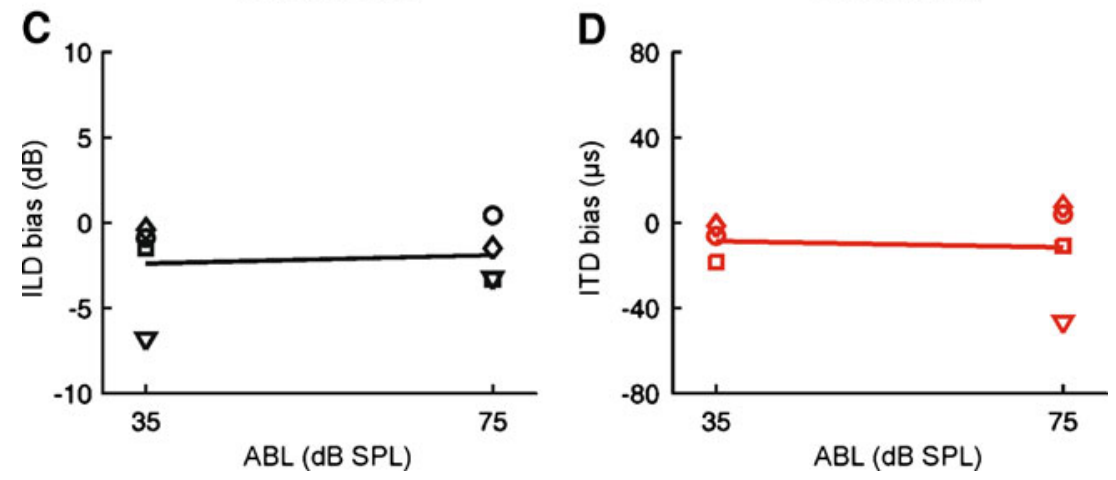

correlation was set to 0.1 , and so these data were excluded from further analysis. For the remaining data, we observed a highly significant effect of interaural correlation on ITD thresholds $(F(1,19)=$ $30.25, P<0.0001)$. Similarly, reducing the interaural correlation had no effect on ILD response bias
$(F(2,33)=0.69, P=0.5099$; Fig. 5C), but did increase ITD response bias $(F(1,19)=7.77, P=0.0117$; Fig. 5D). These data are therefore consistent with ITD processing in ferrets being dependent on a cross-correlation mechanism, whereas this is not the case for ILD processing.
A
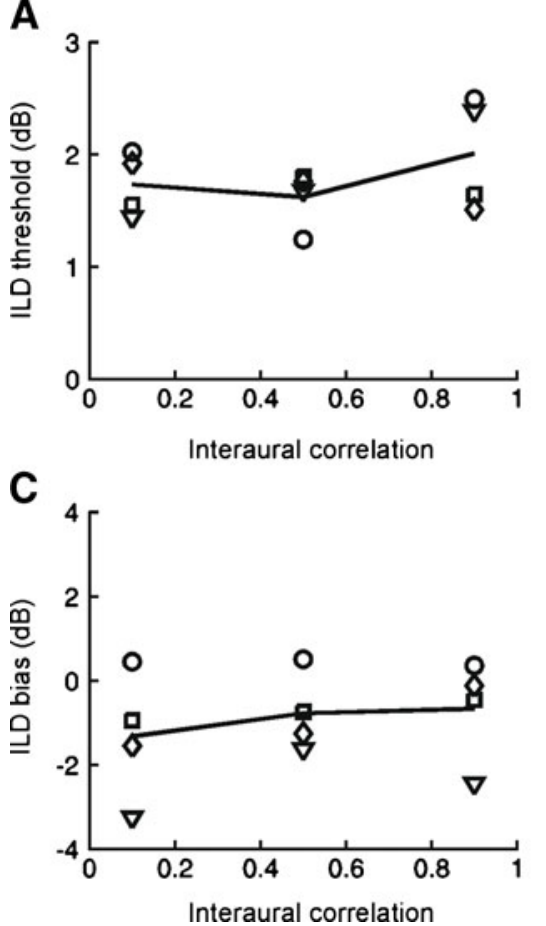

B

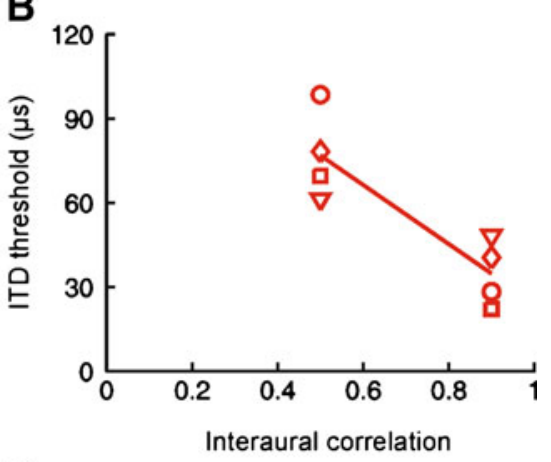

D

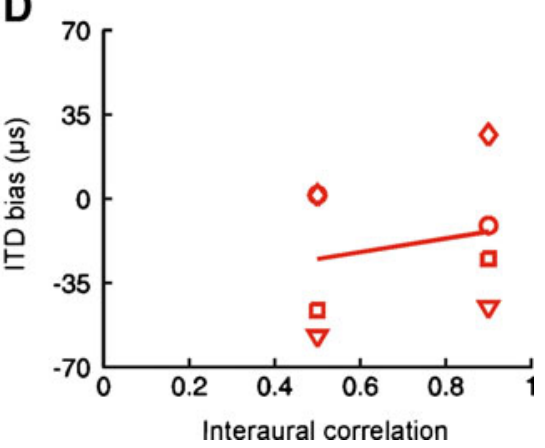

FIG. 5. Effect of interaural correlation on ILD and ITD sensitivity. Symbols show data for individual animals, with lines showing the mean across subjects. A Effect of interaural correlation on ILD thresholds. B Effect of interaural correlation on ITD thresholds. C Effect of interaural correlation on ILD bias. D Effect of interaural correlation on ITD bias. 


\section{DISCUSSION}

Although the sound localization abilities of ferrets have been investigated extensively using free-field stimuli (Kavanagh and Kelly 1987; Parsons et al. 1999; Kacelnik et al. 2006; Nodal et al. 2008), estimates of ITD and ILD sensitivity have so far been lacking. Consistent with closed-field studies in other species, we found that ferrets can detect very small changes in either ITD or ILD, which can account for many aspects of their localization behavior.

\section{Binaural cue sensitivity}

In some cases, ferrets exhibited ITD thresholds of $<20 \mu \mathrm{s}$, with the mean threshold across animals and sessions equal to 23 us for 200-ms broadband stimuli. Despite some differences in methodology between studies, these data are broadly comparable with ITD discrimination thresholds in humans, which are typically 10-20 $\mu$ s (Zwislocki and Feldman 1956; Klumpp and Eady 1957; Yost 1974), as well as those reported for macaques (Scott et al. 2007), cats (Wakeford and Robinson 1974), and owls (Moiseff and Konishi 1981). The ITD thresholds obtained from ferrets are slightly better than those found in rabbits (Ebert et al. 2008), which is consistent with the notion that predatory species may have more developed sound localization abilities. Ferrets are also very sensitive to changes in ILDs, with some animals having thresholds of $<1 \mathrm{~dB}$, while the mean value for $200 \mathrm{~ms}$ of flat-envelope stimuli was $1.3 \mathrm{~dB}$. Again, these thresholds are broadly comparable with those observed in humans, which typically vary from 0.5 to $1 \mathrm{~dB}$ over a wide range of frequencies (Mills 1960), as well as those obtained from macaque monkeys (Scott et al. 2007) and cats (Wakeford and Robinson 1974).

One potential issue for studies of animal behavior is that subjects typically require extensive training on the task prior to testing, which means that thresholds may differ from those that would be observed in experimentally naive animals. We therefore initially trained our ferrets using large ILD values that we knew to be far above naïve thresholds based on prior behavioral testing with additional animals (Nodal et al. 2010). We were thus able to estimate binaural cue sensitivity in animals that had minimal prior exposure to the small ITDs and ILDs used to estimate thresholds. Despite their lack of experience with the perceptually challenging aspects of the task, we found no improvement in behavioral performance over relatively long periods of testing, indicating that the thresholds measured with broadband noise stimuli are stable. Repeated measurements over the course of a few weeks have also produced consistent estimates of sound localization accuracy in ferrets (Smith et al.
2004). It is possible, however, that improvements would be seen had we employed explicit training procedures or used narrowband stimuli that are more difficult to localize, as has been done in humans (Wright and Fitzgerald 2001; Rowan and Lutman 2007; Kumpik et al. 2009).

Free-field studies have shown that ferrets can discriminate between broadband sounds that are separated by $\sim 10^{\circ}$ in azimuth around the midline (Kavanagh and Kelly 1987; King and Parsons 1999), where the minimum audible angle (MAA) was defined as the spatial separation that elicited correct responses on $75 \%$ of trials. To convert the ITD thresholds into equivalent estimates of MAA, we would therefore need to double each threshold and convert from microseconds into degrees. Assuming that the physiological ITD range is approximately $\pm 240 \mu$ s in the ferret (Schnupp et al. 2003), an ITD threshold of $23 \mu$ s would correspond to an MAA of $\sim 17^{\circ}$.

Doing the same analysis for ILDs is complicated by the frequency dependence of these cues under freefield conditions (Carlile 1990; Carlile and King 1994). This frequency dependence occurs because the directional filtering effects of the head and ears, as measured by the head-related transfer function, typically vary as a function of frequency. Consequently, the same MAA will equate to different ILDs at different frequencies. If spatial sensitivity were determined by the frequency-averaged ILD, this would be relatively unproblematic, but because changes in the position of a sound source produce much larger changes in ILD at higher frequencies, it is likely that some frequency bands will confer greater spatial sensitivity than others under free-field conditions. These considerations therefore make it difficult to convert a given MAA into an equivalent ILD threshold.

Nevertheless, if we assume that ferrets experience an ILD range of $\pm 20 \mathrm{~dB}$ in a frequency band for which they have an ILD threshold of $1.3 \mathrm{~dB}$, this would generate an equivalent MAA value of $12^{\circ}$, very close to the previous MAA estimates. However, because ILDs in the ferret are $\geq 20 \mathrm{~dB}$ only over a narrow frequency range when sounds are presented in the free field (Schnupp et al. 2003), this analysis is best interpreted as placing an upper bound on what might be achieved by the ferret auditory system. Thus, it is unlikely that ILD sensitivity alone can account for the levels of spatial acuity that are observed in the free field.

In the free field, the head-related transfer function ensures that ferrets also have access to monaural spectral cues (Carlile 1990; Carlile and King 1994). However, whilst these cues are critical for judging the elevation of a sound (Musicant and Butler 1984), they typically play a negligible role when sound sources are localized in azimuth (Macpherson and Middlebrooks 2002), other than 
for distinguishing between sources located in front of and behind the listener (Carlile and King 1994; Carlile et al. 2005). Our results therefore suggest that previous free-field estimates of MAA for broadband stimuli most likely reflect the combined operation of both ITD and ILD mechanisms.

Although this study represents the first attempt to characterize how well ferrets can discriminate changes in ITDs and ILDs, previous neurophysiological studies in this species have shown that neurons in both the inferior colliculus (IC) (Dahmen et al. 2010) and auditory cortex (Campbell et al. 2006; Hartley et al. 2011) are sensitive to binaural spatial cues. It is difficult, however, to relate these recording data to our behavioral thresholds since no attempt was made to obtain neurometric measures of spatial cue discrimination. Our results therefore highlight the need for future studies of this kind.

Ideally, neurometric measures would be obtained from the responses of cells whilst animals are performing a behavioral task. These techniques have been successfully applied to a spatial discrimination task in macaque monkeys (Recanzone et al. 2000), while Bala et al. (2003) found that the ability of barn owls to detect a change in sound source location can be predicted from the population activity of neurons recorded under anesthesia in the space-mapped region of the IC. Although Skottun et al. (2001) showed that ITD discrimination thresholds estimated for individual neurons in the anesthetized guinea pig IC can be as small as those reported for human listeners, we are unaware of any studies that have attempted to obtain simultaneous estimates of behavioral and neural sensitivity to individual spatial cues. By establishing an appropriate lateralization task for the ferret, this study therefore takes an important step in that direction, paving the way for experiments that combine behavioral and electrophysiological recording techniques in the same animal.

\section{Effects of varying stimulus parameters}

Consistent with previous measurements of both sound localization accuracy (Nodal et al. 2008) and MAAs (Kavanagh and Kelly 1987; King and Parsons 1999) in the ferret, we found that the best thresholds were elicited by stimuli with the longest duration. We observed similar effects of stimulus duration for both ITD and ILD and for different stimulus types, suggesting that this may be a general feature of sound localization in this species, possibly related to the time needed to process the cues or to adaptation effects (Macpherson and Middlebrooks 2000).

In contrast to the dependence of ITD and ILD threshold on stimulus duration, binaural spatial sensitivity in the ferret is relatively robust to changes in the envelope of broadband stimuli, with ITD thresholds showing a slight increase and ILD thresholds remaining the same when SAM was added to the envelope. Because SAM provides ongoing ITD information in the amplitude envelope at high carrier frequencies, we might have expected this to improve ITD sensitivity. A possible explanation for this result is that amplitude modulation interferes with neuronal sensitivity to fine-structure ITDs at low frequencies. Consistent with this view, electrophysiological recordings have shown that amplitude modulation can alter the sensitivity of low-frequency cells in the IC to finestructure ITDs (Sterbing et al. 2003). These changes were not consistently observed, however, and ITD sensitivity at the population level was preserved. The likely consequences at a behavioral level are thus unclear.

We also found that ILD sensitivity was relatively unaffected by sound level, which parallels results from studies of binaural processing in both humans (Dreyer and Oxenham 2008; Kumpik et al. 2010) and cats (Tsai et al. 2010), as well as estimates of freefield localization accuracy in ferrets (Nodal et al. 2008). In contrast, ITD thresholds were larger, and could not always be obtained, at the lowest sound level tested. Given the shape of the ferret audiogram (Kelly et al. 1986), however, as well as the fact that spectrally flat sounds contain less energy per octave at lower frequencies, it is possible that low-frequency inputs were barely audible by the animals when stimuli were presented at $35 \mathrm{~dB}$ SPL. To the extent that low-frequency inputs are particularly important for ITD sensitivity, this could account for the apparent differences between ITD and ILD processing at quiet sound levels.

In comparison with the relatively small effects of most other stimulus parameters, we found that ITD sensitivity is progressively impaired as binaural inputs are decorrelated, with ferrets completely unable to perform the task when interaural correlation was set to low values. This is in line with earlier work in both humans (Blauert and Lindemann 1986) and barn owls (Saberi et al. 1998). In contrast with its effect on ITD processing, changes in interaural correlation had very little effect on ILD sensitivity, a finding that has previously been reported in the barn owl (Egnor 2001). This is consistent with the notion that estimates of ITD, but not ILD, depend on a cross-correlation mechanism (Fischer et al. 2008).

Overall, our results show that behavioral estimates of binaural sensitivity can be readily obtained in the ferret and that this species possesses relatively precise and robust sensitivity to broadband ITDs and ILDs. The thresholds obtained, as well as the effects of different acoustical parameters on binaural processing, are broadly consistent with data obtained in other species. Together, these results confirm the ferret as 
an excellent model for studying spatial hearing and provide important baseline measurements for future studies of auditory processing and plasticity.

\section{ACKNOWLEDGMENTS}

This work was supported by the Wellcome Trust through a Principal Research Fellowship (WT076508AIA) to A.J.K. and by a Newton Abraham Studentship to P.K. We are grateful to Sue Spires, Dan Kumpik, Victoria Bajo, and Sandra Tolnai for their contributions to behavioral testing.

Open Access This article is distributed under the terms of the Creative Commons Attribution License which permits any use, distribution, and reproduction in any medium, provided the original author(s) and the source are credited.

\section{REFERENCES}

Alves-Pinto A, Sollini J, Sumner CJ (2012) Signal detection in animal psychoacoustics: analysis and simulation of sensory and decision-related influences. Neuroscience 220:215-227

Bala AD, Spttzer MW, Takahashi TT (2003) Prediction of auditory spatial acuity from neural images on the owl's auditory space map. Nature 424:771-774

BeE MA, Micheyl C (2008) The cocktail party problem: what is it? How can it be solved? And why should animal behaviorists study it? J Comp Psychol 122:235-251

Bizley JK, Nodal FR, Parsons CH, King AJ (2007) Role of auditory cortex in sound localization in the midsagittal plane. J Neurophysiol 98:1763-1774

Bizley JK, Walker KMM, King AJ, Schnupp JW (2013) Spectral timbre perception in ferrets: discrimination of artificial vowels under different listening conditions. J Acoust Soc Am 133:365-376

BLAuert J, Lindemann W (1986) Spatial mapping of intracranial auditory events for various degrees of interaural coherence. J Acoust Soc Am 79:806-813

Campbell RA, Schnupp JW, Shial A, King AJ (2006) Binaural-level functions in ferret auditory cortex: evidence for a continuous distribution of response properties. J Neurophysiol 95:37423755

CARLILE S (1990) The auditory periphery of the ferret. I: directional response properties and the pattern of interaural level differences. J Acoust Soc Am 88:2180-2195

Carlile S, King AJ (1994) Monaural and binaural spectrum level cues in the ferret: acoustics and the neural representation of auditory space. J Neurophysiol 71:785-801

Carlile S, Martin R, McAnally K (2005) Spectral information in sound localization. Int Rev Neurobiol 70:399-434

Culling JF, Colburn HS, Spurchise M (2001) Interaural correlation sensitivity. J Acoust Soc Am 110:1020-1029

Dahmen JC, Keating P, Nodal FR, Schulz AL, King AJ (2010) Adaptation to stimulus statistics in the perception and neural representation of auditory space. Neuron 66:937-948

Dreyer AA, Oxenham AJ (2008) Effects of level and background noise on interaural time difference discrimination for transposed stimuli. J Acoust Soc Am 123:EL1-EL7

Ebert CS Jr, Blanks DA, Patel Mr, Coffey CS, Marshall aF, FitzPATRICK DC (2008) Behavioral sensitivity to interaural time differences in the rabbit. Hear Res 235:134-142
EGNOR SE (2001) Effects of binaural decorrelation on neural and behavioral processing of interaural level differences in the barn owl (Tyto alba). J Comp Physiol A 187:589-595

Fischer BJ, Christianson GB, Pena JL (2008) Cross-correlation in the auditory coincidence detectors of owls. J Neurosci 28:8107-8115

Green DM, Swets JA (1966) Signal detection theory and psychophysics. Wiley, New York

Hartley DE, Dahmen JC, King AJ, Schnupp JW (2011) Binaural sensitivity changes between cortical on and off responses. J Neurophysiol 106:30-43

Hartley De, Vongpaisal T, Xu J, Shepherd RK, King AJ, Isaiah A (2010) Bilateral cochlear implantation in the ferret: a novel animal model for behavioral studies. J Neurosci Methods 190:214-228

Hine JE, Martin RL, Moore DR (1994) Free-field binaural unmasking in ferrets. Behav Neurosci 108:196-205

Kacelnik O, Nodal FR, Parsons CH, King AJ (2006) Traininginduced plasticity of auditory localization in adult mammals. PLoS Biol 4:627-638

Kavanagh GL, Kelly JB (1987) Contribution of auditory cortex to sound localization by the ferret (Mustela putorius). J Neurophysiol 57:1746-1766

Kelly JB, Kavanagh GL, Dalton JC (1986) Hearing in the ferret (Mustela putorius): thresholds for pure tone detection. Hear Res 24:269-275

Kelly JB, Rooney BJ, Phillips DP (1996) Effects of bilateral auditory cortical lesions on gap-detection thresholds in the ferret (Mustela putorius). Behav Neurosci 110:542-550

King AJ, Parsons CH (1999) Improved auditory spatial acuity in visually deprived ferrets. Eur J Neurosci 11:3945-3956

King AJ, Dahmen JC, Keating P, Leach ND, Nodal FR, Bajo VM (2011) Neural circuits underlying adaptation and learning in the perception of auditory space. Neurosci Biobehav Rev 35:2129-2139

KLumpP RG, EAdY HR (1957) Some measurements of interaural time difference thresholds. J Acoust Soc Am 28:859-860

Kumpik D, Ting J, Campbell RA, Schnupp JW, King AJ (2009) Specificity of binaural perceptual learning for amplitude modulated tones: a comparison of two training methods. J Acoust Soc Am 125:2221-2232

Kumpik DP, Kacelnik O, KIng AJ (2010) Adaptive reweighting of auditory localization cues in response to chronic unilateral earplugging in humans. J Neurosci 30:4883-4894

MacPherson EA, Middebbrooks JC (2000) Localization of brief sounds: effects of level and background noise. J Acoust Soc Am 108:1834-1849

MacPherson EA, Middlebrooks JC (2002) Listener weighting of cues for lateral angle: the duplex theory of sound localization revisited. J Acoust Soc Am 111:2219-2236

Mills AW (1960) Lateralization of high-frequency tones. J Acoust Soc Am 32:132-134

MoIsefF A, Konishi M (1981) Neuronal and behavioral sensitivity to binaural time differences in the owl. J Neurosci 1:40-48

MoOre DR (2002) Auditory development and the role of experience. Br Med Bull 63:171-181

Musicant AD, Butler RA (1984) The influence of pinnae-based spectral cues on sound localization. J Acoust Soc Am 75:11951200

Nodal FR, Keating P, King AJ (2010) Chronic detachable headphones for acoustic stimulation in freely moving animals. J Neurosci Methods 189:44-50

Nodal FR, Bajo VM, Parsons CH, Schnupp JW, King AJ (2008) Sound localization behavior in ferrets: comparison of acoustic orientation and approach-to-target responses. Neuroscience 154:397408

Parsons CH, Lanyon RG, Schnupp JW, King AJ (1999) Effects of altering spectral cues in infancy on horizontal and vertical sound localization by adult ferrets. J Neurophysiol 82:2294-2309 
PeÑa JL (2003) Binaural processing in the synthesis of auditory spatial receptive fields. Biol Cybern 89:371-377

Recanzone GH, Guard DC, Phan ML, Su TK (2000) Correlation between the activity of single auditory cortical neurons and sound-localization behavior in the macaque monkey. J Neurophysiol 83:2723-2739

Roman N, Wang D, Brown GJ (2003) Speech segregation based on sound localization. J Acoust Soc Am 114:2236-2252

Rowan D, Lutman ME (2007) Learning to discriminate interaural time differences at low and high frequencies. Int J Audiol 46:585-594

Saberi K, Takahashi Y, Konishi M, Albeck Y, Arthur BJ, Farahbod H (1998) Effects of interaural decorrelation on neural and behavioral detection of spatial cues. Neuron 21:789-798

Schnupp JW, Boоth J, KIng AJ (2003) Modeling individual differences in ferret external ear transfer functions. J Acoust Soc Am 113:2021-2030

Scott BH, Malone BJ, Semple MN (2007) Effect of behavioral context on representation of a spatial cue in core auditory cortex of awake macaques. J Neurosci 27:6489-6499

Skottun BC, Shackleton TM, Arnott RH, Palmer AR (2001) The ability of inferior colliculus neurons to signal differences in interaural delay. Proc Natl Acad Sci U S A 98:14050-14054

Smith AL, Parsons CH, Lanyon RG, Bizley JK, Akerman CJ, Baker GE, Dempster AC, Thompson ID, King AJ (2004) An investigation of the role of auditory cortex in sound localization using muscimolreleasing Elvax. Eur J Neurosci 19:3059-3072

Sterbing SJ, D'Angelo WR, Ostapoff EM, Kunada S (2003) Effects of amplitude modulation on the coding of interaural time differences of low-frequency sounds in the inferior colliculus. I. Response properties. J Neurophysiol 90:2818-2826

Tsai JJ, KoKa K, TolLin DJ (2010) Varying overall sound intensity to the two ears impacts interaural level difference discrimination thresholds by single neurons in the lateral superior olive. J Neurophysiol 103:875-886

WAKEFORD OS, ROBINSON DE (1974) Lateralization of tonal stimuli by the cat. J Acoust Soc Am 55:649-652

Walker KM, Schnupp JW, Hart-Schnupp SM, King AJ, Bizley JK (2009) Pitch discrimination by ferrets for simple and complex sounds. J Acoust Soc Am 126:1321-1335

WitTen IB, KNudSen PF, Knudsen EI (2010) A dominance hierarchy of auditory spatial cues in barn owls. PLoS One 5:e10396

Wright BA, FitzGerald MB (2001) Different patterns of human discrimination learning for two interaural cues to sound-source location. Proc Natl Acad Sci U S A 98:12307-12312

Yin P, Fritz JB, Shamma SA (2010) Do ferrets perceive relative pitch? J Acoust Soc Am 127:1673-1680

Yost WA (1974) Discriminations of interaural phase differences. J Acoust Soc Am 55:1299-1303

ZwISLOCKI J, FeLdman R (1956) Just noticeable difference in dichotic phase. J Acoust Soc Am 28:860-864 